\title{
Explicit symplectic integrators for solving non-separable Hamiltonians.
}

\author{
Siu A. Chin \\ Department of Physics, Texas AEMM University, College Station, TX 77843, USA
}

\begin{abstract}
By exploiting the error functions of explicit symplectic integrators for solving separable Hamiltonians, I show that it is possible to develop explicit, time-reversible symplectic integrators for solving non-separable Hamiltonians of the product form. The algorithms are unusual in that they are of fractional orders.
\end{abstract}

\section{INTRODUCTION}

Symplectic integrators ${ }^{1-4}$ are the methods of choice for solving diverse physical problems in classical $^{1,5-7}$, quantum $^{8-15}$, and statistical ${ }^{16-19}$ mechanics. For separable Hamiltonians, the problem is well understood and many explicit integrators are available ${ }^{1-4}$. However, for non-separable Hamiltonians, only implicit algorithms are known ${ }^{1-4}$. It is generally believed that no explicit algorithms can be developed for solving non-separable Hamiltonians ${ }^{1,2}$. In this work, I show that this is not the case. Explicit, time-reversible algorithms can be developed to solve a selected class of non-separable Hamiltonians. The idea is to model non-separable Hamiltonians by the error terms of explicit algorithms when solving separable Hamiltonians. By a suitable choice of factorization (or split) coefficients, the explicit algorithm can be made to solve error Hamiltonians which are generally non-separable.

In the usual study of symplectic integratiors, one seeks to eliminate error terms in order to produce higher order algorithms. These error terms are therefore not of direct interest and are rarely studied in their own right. In this work, these error terms are the non-separable Hamiltonians we seek to solve. The method can solve non-separable Hamiltonians of the product form, (sum over repeated indices)

$$
H=T_{i}(\mathbf{p}) V_{i j}(\mathbf{q}) T_{i}(\mathbf{p}),
$$

provided that

$$
T_{i}(\mathbf{p})=\frac{\partial}{\partial p_{i}} T(\mathbf{p})
$$

and

$$
V_{i j}(\mathbf{q})=\frac{\partial^{2}}{\partial q_{i} \partial q_{j}} V(\mathbf{q}) .
$$

For one degree of freedom, given $T^{\prime}(p)$ and $V^{\prime \prime}(q), T(p)$ and $V(q)$ can always be obtained by integration.

In the next section we will briefly summarize essential aspects of symplectic integrators and their error functions, followed by our explicit integrator for solving the above non-separable Hamiltonian. Higher order algorithms are discussed in Section IV.

\section{SYMPLECTIC INTEGRATORS}

Given a dynamical variable $W\left(q_{i}, p_{i}\right)$ and a Hamiltonian function $H\left(q_{i}, p_{i}\right)$, the former is evolved by the later via the Poisson bracket, and therefore by the corresponding Lie operator ${ }^{20} \hat{H}$ associated with the function $H\left(q_{i}, p_{i}\right)$,

$$
\begin{aligned}
\frac{d W}{d t} & =\{W, H\} \\
& =\left(\frac{\partial H}{\partial p_{i}} \frac{\partial}{\partial q_{i}}-\frac{\partial H}{\partial q_{i}} \frac{\partial}{\partial p_{i}}\right) W=\hat{H} W,
\end{aligned}
$$

via exponentiation,

$$
W(t+\varepsilon)=\mathrm{e}^{\varepsilon \hat{H}} W(t) .
$$

For a separable Hamiltonian,

$$
H(\mathbf{q}, \mathbf{p})=T(\mathbf{p})+V(\mathbf{q}),
$$


the corresponding Hamiltonian operator is also separable, $\hat{H}=\hat{T}+\hat{V}$, with $\hat{T}$ and $\hat{V}$ given by

$$
\begin{gathered}
\hat{T} \equiv\{\cdot, T\}=\frac{\partial T}{\partial p_{i}} \frac{\partial}{\partial q_{i}}, \\
\hat{V} \equiv\{\cdot, V\}=-\frac{\partial V}{\partial q_{i}} \frac{\partial}{\partial p_{i}} .
\end{gathered}
$$

Their corresponding evolution operators $\mathrm{e}^{\varepsilon \hat{T}}$ and $\mathrm{e}^{\varepsilon \hat{V}}$ then shift $q_{i}$ and $p_{i}$ forward in time via

$$
\begin{aligned}
& q_{i}(\varepsilon)=\mathrm{e}^{\varepsilon \hat{T}} q_{i}=q_{i}+\varepsilon \frac{\partial T}{\partial p_{i}} \\
& p_{i}(\varepsilon)=\mathrm{e}^{\varepsilon \hat{V}} p_{i}=p_{i}-\varepsilon \frac{\partial V}{\partial q_{i}} .
\end{aligned}
$$

Conventional symplectic integrators correspond to approximating the short time evolution operator $\mathrm{e}^{\varepsilon \hat{H}}$ in the product form

$$
\mathrm{e}^{\varepsilon(\hat{T}+\hat{V})} \approx \prod_{i=1}^{N} \mathrm{e}^{t_{i} \varepsilon \hat{T}} \mathrm{e}^{v_{i} \varepsilon \hat{V}}
$$

resulting in an ordered sequence of displacements (9) which defines the resulting algorithm. Here, we will consider only time-reversible, symmetric factorization schemes such that either $t_{1}=0$ and $v_{i}=v_{N-i+1}, t_{i+1}=t_{N-i+1}$, or $v_{N}=0$ and $v_{i}=v_{N-i}, t_{i}=t_{N-i+1}$.

The product of operators in (10) can be combined by use of the Baker-Campbell-Hausdorff (BCH) formula to give

$$
\prod_{i=1}^{N} \mathrm{e}^{t_{i} \varepsilon \hat{T}} \mathrm{e}^{v_{i} \varepsilon \hat{V}}=\mathrm{e}^{\varepsilon \hat{H}_{A}},
$$

where the approximate Hamiltonian operator $\hat{H}_{A}$ has the general form

$$
\begin{gathered}
\hat{H}_{A}=e_{T} \hat{T}+e_{V} \hat{V}+\varepsilon^{2} e_{T T V}[\hat{T} \hat{T} \hat{V}] \\
+\varepsilon^{2} e_{V T V}[\hat{V} \hat{T} \hat{V}]+O\left(\varepsilon^{4}\right)
\end{gathered}
$$

where $e_{T}, e_{T V}, e_{T T V}$, etc., are functions of $\left\{t_{i}\right\}$ and $\left\{v_{i}\right\}$ and where condensed commutator brackets, $[\hat{T} \hat{T} \hat{V}]=$ $[\hat{T},[\hat{T}, \hat{V}]],[\hat{T} \hat{V} \hat{T} \hat{V}]=[\hat{T},[\hat{V},[\hat{T}, \hat{V}]]]$, etc., are used. From the way Lie operators are defined via (4), one can convert operators back to functions ${ }^{1,7}$ via $[T, V] \rightarrow\{V, T\}=-\{T, V\}$, yielding

$$
\begin{gathered}
H_{A}=e_{T} T+e_{V} V+\varepsilon^{2} e_{T T V}\{T T V\} \\
+\varepsilon^{2} e_{V T V}\{V T V\}+O\left(\varepsilon^{4}\right),
\end{gathered}
$$

where again, condensed Poisson brackets, $\{T T V\}=\{T,\{T, V\}\}$, etc., are used. For a separable Hamiltonian of the form (6), we have

$$
\begin{aligned}
\{T V\} & =-\frac{\partial T}{\partial p_{j}} \frac{\partial V}{\partial q_{j}}=-T_{j} V_{j}, \\
\{T T V\} & =-\frac{\partial T}{\partial p_{i}} \frac{\partial\{T, V\}}{\partial q_{i}}=T_{i} V_{i j} T_{j}, \\
\{V T V\} & =\frac{\partial V}{\partial q_{i}} \frac{\partial\{T, V\}}{\partial p_{i}}=-V_{i} T_{i j} V_{j} .
\end{aligned}
$$

By choosing $\left\{t_{i}\right\}$ and $\left\{v_{i}\right\}$ such that

$$
e_{T}=e_{V}=0
$$

and either $e_{V T V}=0$, or $e_{T T V}=0$, the algorithm would then be solving the non-separable Hamiltonian, either

$$
H_{T T V}=T_{i} V_{i j} T_{j} \quad \text { or } \quad H_{V V T}=V_{i} T_{i j} V_{j}
$$




\section{SOLVING NON-SEPARABLE HAMILTONIANS}

The following factorization scheme gives,

$$
\begin{aligned}
\mathcal{T}(\varepsilon) & \equiv \mathrm{e}^{\varepsilon v_{2} \hat{V}} \mathrm{e}^{\varepsilon t_{2} \hat{T}} \mathrm{e}^{\varepsilon v_{1} \hat{V}} \mathrm{e}^{\varepsilon t_{1} \hat{T}} \mathrm{e}^{\varepsilon v_{0} \hat{V}} \mathrm{e}^{\varepsilon t_{1} \hat{T}} \mathrm{e}^{\varepsilon v_{1} \hat{V}} \mathrm{e}^{\varepsilon t_{2} \hat{T}} \mathrm{e}^{\varepsilon v_{2} \hat{V}} \\
& =\exp \left(\varepsilon^{3}[\hat{T} \hat{T} \hat{V}]+\varepsilon^{5} E_{5}+\varepsilon^{7} E_{7}+\varepsilon^{9} E_{9} \cdots\right),
\end{aligned}
$$

with $v_{0}=-2\left(v_{1}+v_{2}\right), t_{1}=-t_{2}, v_{2}=-v_{1} / 2$ and $v_{1}=1 / t_{2}^{2}$. There is one free parameter $t_{2}$ that one can choose to minimize the resulting error, but not be set to zero. As examplfied by (14) and (15), for a separable Hamiltonian $H=T+V$, higher order brackets of the form $\{T, Q\},\{V, Q\}$ have opposite signs. Thus one should choose algorithms with $e_{T Q}=e_{V Q}$ to maximize error cancellations ${ }^{19}$. This is the basis for symplectic corrector ${ }^{21}$ or processed ${ }^{22,23}$ algorithms. The choice of $t_{2}=-6^{1 / 3} \approx-1.82$ forces $e_{T T T T V}=e_{V T T T V}$ and would be a good starting value. The RHS of (18) is the evolution operator for the non-separable Hamiltonian $H_{T T V}$ with time step $\Delta t=\varepsilon^{3}$ and leading error terms $O\left(\varepsilon^{5}\right)$. Thus the parameter $\varepsilon$ used by the integrator is $\varepsilon=\sqrt[3]{\Delta t}$. Since $\varepsilon^{5}=\Delta t^{5 / 3}$, the basic algorithm (18) in terms of $\Delta t$ reads,

$$
\mathcal{T}(\Delta t)=\exp \Delta t\left([\hat{T} \hat{T} \hat{V}]+\Delta t^{2 / 3} E_{5}+\Delta t^{4 / 3} E_{7}+\Delta t^{6 / 3} E_{9} \cdots\right) .
$$

The order of the algorithm $\mathcal{T}(\Delta t)$ (the leading error in the Hamiltonian) is therefore only $2 / 3$. We will discuss this and higher order algorithms in the next section.

By interchange $\hat{T} \leftrightarrow \hat{V}$ everywhere, but keeping the coefficents intact, the RHS of (18) goes over to

$$
\mathrm{e}^{\varepsilon^{3}[\hat{T} \hat{T} \hat{V}]} \rightarrow \mathrm{e}^{\varepsilon^{3}[\hat{V} \hat{V} \hat{T}]}
$$

and the basica algorithm $\mathcal{T}(\Delta t)$ solves the non-separable Hamiltonian $H_{V V T}$. In both cases, the final force or velocity can be re-used at the start of the next iteration. Thus both algorithms require four-force and four-velocity evaluations.

For one degree of freedom, any Hamiltonian of the form

$$
H=f(p) g(q)
$$

can be solved. To test the algorithm, we solve the non-separable Hamiltonian

$$
H_{T T V}=\left(1+\frac{p^{2}}{2}\right)^{2}\left(1+q^{2}\right)
$$

where the phase trajectory is harmonic near the origin, but highly distorted at larger values of $(p, q)$. The algorithm's separable Hamiltonian is

$$
H=p+\frac{1}{6} p^{3}+\frac{1}{2} q^{2}+\frac{1}{12} q^{4}
$$

In Fig.1 we compare the phase trajectories produced by algorithm (18) with exact trajectories deduced from (22). We set $t_{2}=-2$ and use a relatively large value of $\Delta t=0.005$ so that discrepances can be seen. The four trajectories are started at $p_{0}=0$ and $q_{0}=0.5,1.0,1.5$, and 2.0 respectively. The error is largest at the positive maximum of $p$ and next largest at the negative maximum of $p$. In each case, the error can be further reduced by making $t_{2}$ more negative than -2 . We did not bother with this refinement here, but this will be important in the 2D case discussed below.

We will demonstrate that $\mathcal{T}(\Delta t)$ indeed converges as $\Delta t^{2 / 3}$ in the next section.

For more than one degree of freedom, the generalization of (21) to

$$
H=\sum_{i} f_{i}\left(p_{i}\right) g_{i}\left(q_{i}\right)
$$

can always be solved. However, it is more interesting to generalize (23) to N-dimension by reinterpreting $p$ and $q$ as radial coordinates: $p=\sqrt{\sum_{i} p_{i}^{2}}, q=\sqrt{\sum_{i} q_{i}^{2}}$. For any radial potential $V(q)$,

$$
V_{i j}=\frac{V^{\prime}}{q} \delta_{i j}+\left(V^{\prime \prime}-\frac{V^{\prime}}{q}\right) \hat{q}_{i} \hat{q}_{j}
$$


where here $\hat{\mathbf{q}}$ is the unit vector. Thus the non-separable Hamiltonian $H_{T T V}$ corresponding to the radial Hamiltonian $(23)$ is

$$
H_{T T V}=\left(1+\frac{p^{2}}{2}\right)^{2}\left[1+\frac{1}{3} q^{2}+\frac{2}{3} q^{2}(\hat{\mathbf{p}} \cdot \hat{\mathbf{q}})^{2} .\right]
$$

This can again be solved by our explicit integrator (18). In two-dimension, most trajectories are not closed and are likely to be chaotic. However, for some special initial configurations, a rich variety of closed orbits can be found. Fig. 2 shows a sample of three such closed orbits. For this calculation, since the order of the algorithm is only $2 / 3$, reducing the step size is not efficient in achieving higher accuaracy. Instead, we find that the error can be substantially reduced by changing $t_{2}$ to $\approx-3$. For the circle, triangle and the twisted orbits of Fig. 3 , the step sizes used were, $\Delta t=0.0012$, 0.001 , and 0.0005 respectively.

Finally, the standard kinetic energy term

$$
T(\mathbf{p})=\frac{1}{2} p_{i} p_{i}
$$

produces

$$
\begin{aligned}
& H_{T T V}=\{T T V\}=p_{i} V_{i j} p_{j}, \\
& H_{V T V}=\{V T V\}=-V_{i} V_{i},
\end{aligned}
$$

and only $H_{T T V}$ is non-separable. Here, $V_{i j}$ can be viewed as a position-dependent inverse mass matrix. This work shows that if $V_{i j}$ can be derived from a potential function $V(\mathbf{q})$, then this non-separable Hamiltonian can also be solved by our explicit algorithm. Also, by itself, this quadratic Hamiltonian does not possess closed orbits for most $V(\mathbf{q})$, thus explaining why this error term would disrupt closed orbit of the original Hamiltonian at large $\varepsilon$.

\section{HIGHER ORDER ALGORITHMS}

In the previous section, we have shown that the primitive algorithm $\mathcal{T}(\Delta t)$ does work and reproduces the correct phase trajectory. However, its $2 / 3$-order convergence is very poor and requires extremely small $\Delta t$ to produce accurate results. To demonstrate its fractional order convergence, we return to the one-dimensional case (22) and integrate from $t=0, p_{0}=0, q_{0}=2$ to $t=T_{1 / 4} \equiv 0.385841, p(t)=-1.569196, q(t)=0$, corresponding to a quarter, clockwise rotation of the outermost phase trajectory of Fig.1. In Fig.3, the relative error of the Hamiltonian $(22)$ at $t=T_{1 / 4}$ is plotted as a function of $\Delta t$. The error of $\mathcal{T}(\Delta t)$ can be perfectly fitted with the power law $-2 \Delta t^{2 / 3}$, but due to this fractional power, the convergence at small $\Delta t$ is very poor. Fortunately, the error structure $(19)$ of $\mathcal{T}(\Delta t)$ allows simple ways of generating higher order symplectic algorithms. The triplet-construction of Creutz and Gocksch ${ }^{24}$ and Yoshida $^{25}$ can produce arbitrary high order algorithms such as the following 4/3rd order algorithm

$$
\mathcal{T}_{4 / 3}(\Delta t)=\mathcal{T}\left(\frac{\Delta t}{2-s}\right) \mathcal{T}\left(-\frac{s \Delta t}{2-s}\right) \mathcal{T}\left(\frac{\Delta t}{2-s}\right)
$$

with $s=2^{3 / 5}$ and the following $6 / 3 \mathrm{rd}=2$ nd-order algorithm

$$
\mathcal{T}_{2}(\Delta t)=\mathcal{T}_{4 / 3}\left(\frac{\Delta t}{2-s}\right) \mathcal{T}_{4 / 3}\left(-\frac{s \Delta t}{2-s}\right) \mathcal{T}_{4 / 3}\left(\frac{\Delta t}{2-s}\right)
$$

with $s=2^{3 / 7}$. As can be seen in Fig.3, these higher order symplectic algorithms are orders of magnitude better than the basic algorithm $\mathcal{T}(\Delta t)$. The disadvantage of the triplet-construction is that the computational effort triples in going from order $k / 3$ to $(k+2) / 3$. For example, the second-order algorithm $\mathcal{T}_{2}(\Delta t)$ requires three evaluations of $\mathcal{T}_{4 / 3}(\Delta t)$, or nine evaluations of $\mathcal{T}(\Delta t)$. Alternatively, arbitrary high order algorithms can also be obtained via the Multi-Product Expansion(MPE) ${ }^{26}$, with only quadratically growing computational efforts. For example, by replacing $k_{i}^{2} \rightarrow k_{i}^{2 / 3}$ in $^{26}$, one obtains

$$
\mathcal{T}_{4 / 3}^{M P E}(\Delta t)=\frac{1^{n}}{1^{n}-2^{n}} \mathcal{T}(\Delta t)+\frac{2^{n}}{2^{n}-1^{n}} \mathcal{T}^{2}\left(\frac{\Delta t}{2}\right)
$$




$$
\begin{aligned}
\mathcal{T}_{2}^{M P E}(\Delta t)=\frac{\left(1^{n}\right)^{2}}{\left(1^{n}-2^{n}\right)\left(1^{n}-3^{n}\right)} \mathcal{T}(\Delta t) & +\frac{\left(2^{n}\right)^{2}}{\left(2^{n}-1^{n}\right)\left(2^{n}-3^{n}\right)} \mathcal{T}^{2}\left(\frac{\Delta t}{2}\right) \\
& +\frac{\left(3^{n}\right)^{2}}{\left(3^{n}-1^{n}\right)\left(3^{n}-2^{n}\right)} \mathcal{T}^{3}\left(\frac{\Delta t}{3}\right)
\end{aligned}
$$

with $n=2 / 3$ in both cases. Here, $\mathcal{T}_{2}^{M P E}(\Delta t)$ only requires six evaluations of $\mathcal{T}(\Delta t)$. The disadvantage of MPE is that it is no longer symplectic, but is like Runge-Kutta-Nyström type algorithms. However, as shown in Fig.3, their energy error can be much smaller than the triplet symplectic algorithms.

\section{CONCLUDING SUMMARY}

In this work, we have shown that explicit symplectic integrators can be devised to solve a selected class of nonseparable Hamiltonians. Any non-separable Hamiltonian which can be modelled by the error terms of an explicit integrator can be solved by the same integrator with changed splitting coefficients. The initial explicit algorithm is only of fractional order $\Delta t^{2 / 3}$, but higher order algorithms can be easily obtained by use of the triplet construction or the multi-product expansion.

1 H. Yoshida, Celest. Mech. Dyn. Astron. 56 (1993) 27.

2 R.I. McLachlan and P. Atela, Nonlinearity, 5 (1992) 541.

3 R. I. McLachlan and G. R. W. Quispel, Acta Numerica, 11 (2002) 241.

4 Geometric Numerical Integration, by E. Hairer, C. Lubich, and G. Wanner, Springer-Verlag, Berlin-New York, 2002.

5 R. D. Skeel, G. Zhang, T. Schlick, SIAM J. Sci. Comput. 18 (1997) 203.

6 S. A. Chin, and C. R. Chen, Cele. Mech. Dyn. Astron. 91 (2005) 301

7 S. Scuro and S. A. Chin, Phys. Rev. E 71 (2005) 056703.

8 A. D. Bandrauk and H. Shen, J. Chem. Phys. 99 (1993) 1185.

9 J. M. Sanz-Serna and A. Portillo, J. Chem. Phys. 104 (1996) 2349.

10 S. A. Chin and C. R. Chen, J. Chem. Phys. 114 (2001) 7338.

11 S. A. Chin and C. R. Chin, J. Chem. Phys. 117 (2002) 1409.

12 H. A. Forbert and S. A. Chin, Phys. Rev. B 63 (2001) 144518.

13 J. Auer, E. Krotscheck, and S. A. Chin, J. Chem. Phys. 115 (2001) 6841.

14 O. Ciftja and S. A. Chin, Phys. Rev. B 68 (2003) 134510.

15 S. A. Chin and E. Krotscheck, Phys. Rev. E 72 (2005) 036705.

16 M. Takahashi and M. Imada, J. Phys. Soc. Jpn 53 (1984) 3765.

17 X. P. Li and J. Q. Broughton, J. Chem. Phys. 86 (1987) 5094.

18 S. Jang, S. Jang and G. A. Voth, J. Chem. Phys. 115 (2001) 7832.

19 S. A. Chin, Phys. Rev. E 69 (2004) 046118. Erratum: $e_{T V T}$ should read $e_{V T V}$ everywhere.

20 A. J. Dragt and J. M. Finn, J. Math. Phys. 172215 (1976)

21 J. Wisdom, M. Holman and J. Touma, "Symplectic correctors", in Integration Algorithms and Classical Mechanics, Fields Inst. Commun., 10, Amer. Math. Soc., Providence, RI, 1996.

22 M. A. Lopez-Marcos, J. M. Sanz-Serna, and R. D. Skeel, SIAM J. Sci. Comput., 18 (1997) 223.

23 S. Blanes, F. Casas, and J. Ros, Siam J. Sci. Comput., 21 (1999) 711.

24 M. Creutz and A. Gocksch, Phys. Rev. Letts. 63, 9 (1989).

25 H. Yoshida, Phys. Lett. A150, 262-268.

26 S. A. Chin, "Multi-product splitting and Runge-Kutta-Nystrom integrators", ArXiv:0809.0914. 


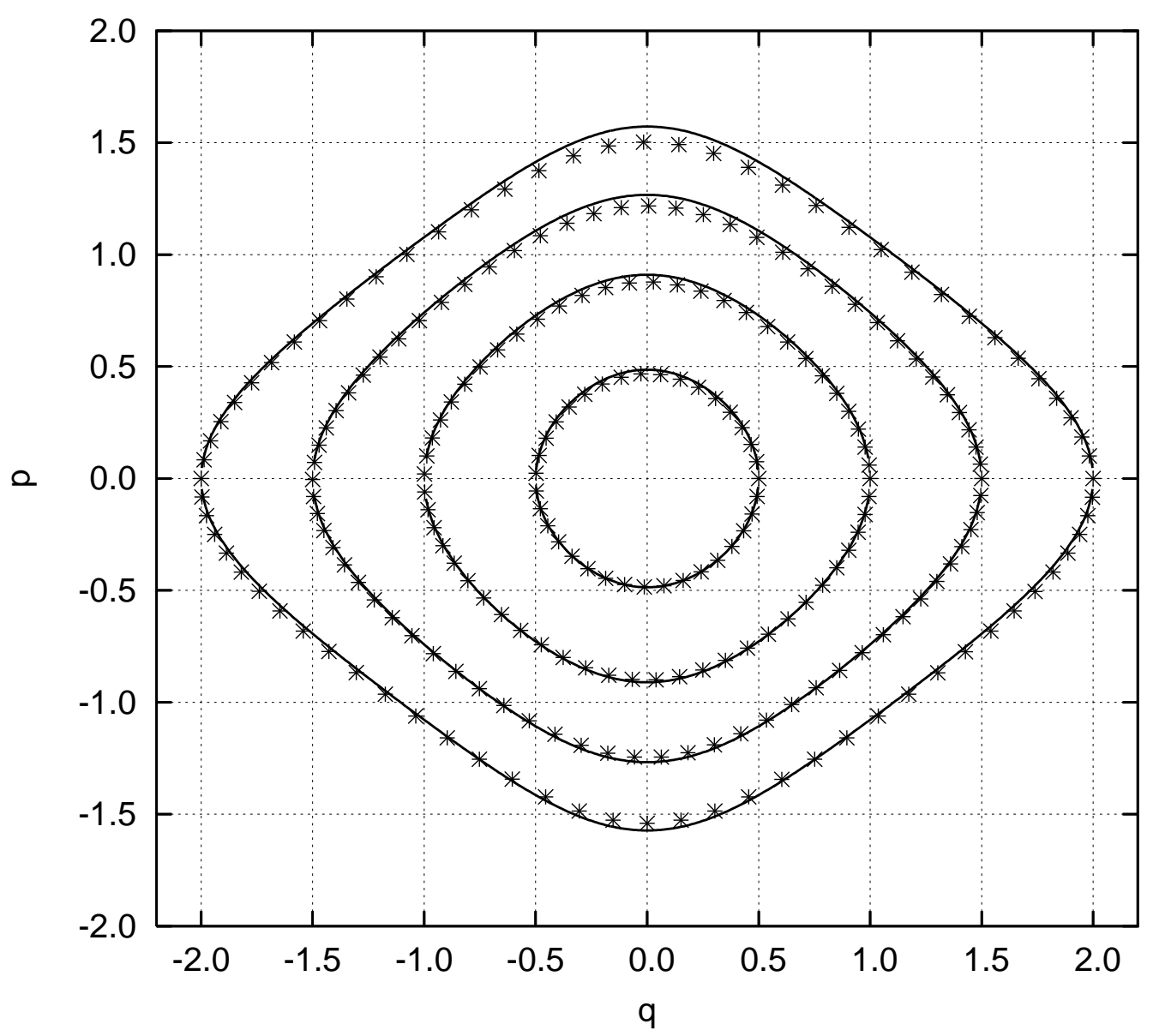

FIG. 1: The phase trajectories of the non-separable Hamiltonian (22). The computed phase points (stars) are compared with exact trajectories (lines). The initial values are $p_{0}=0$ and $q_{0}=0.5,1.0,1.5$ and 2.0 , corresponding to energy values of 1.25 , $2.0,3.25$ and 5.0 respectively. 


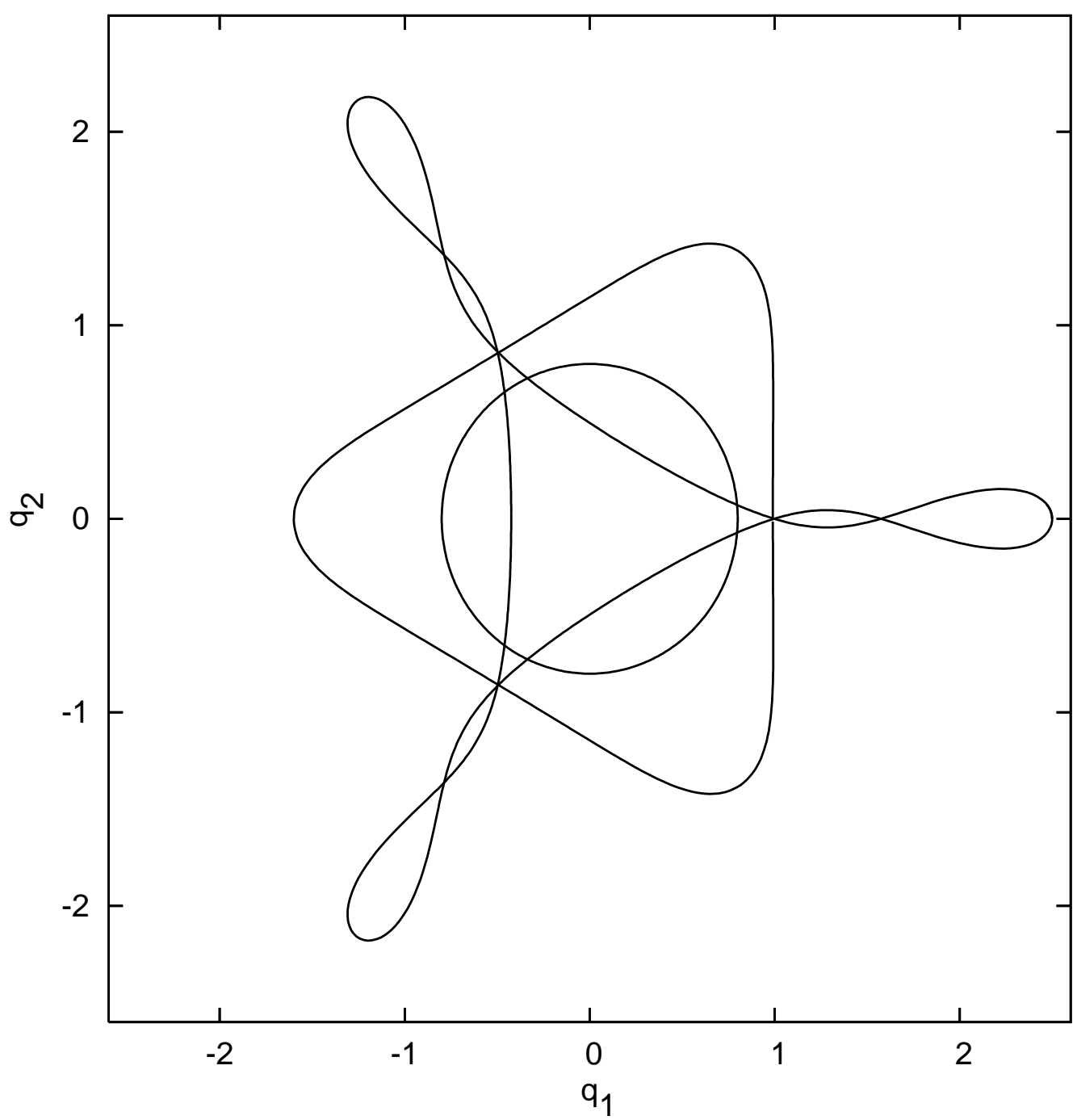

FIG. 2: Some two dimensional orbits of the non-separable Hamiltonian (26). Most trajectory are not closed and only very special initial conditions can result in closed orbits. The initial conditions $\left(q_{1}, q_{2}, p_{1}, p_{2}\right)$ that produce the circle, the triangle and the twisted orbits are respectively, $(0.8,0,0,0.425),(0.99,0,0,0.789)$ and $(2.5,0,0,0.1884)$. 


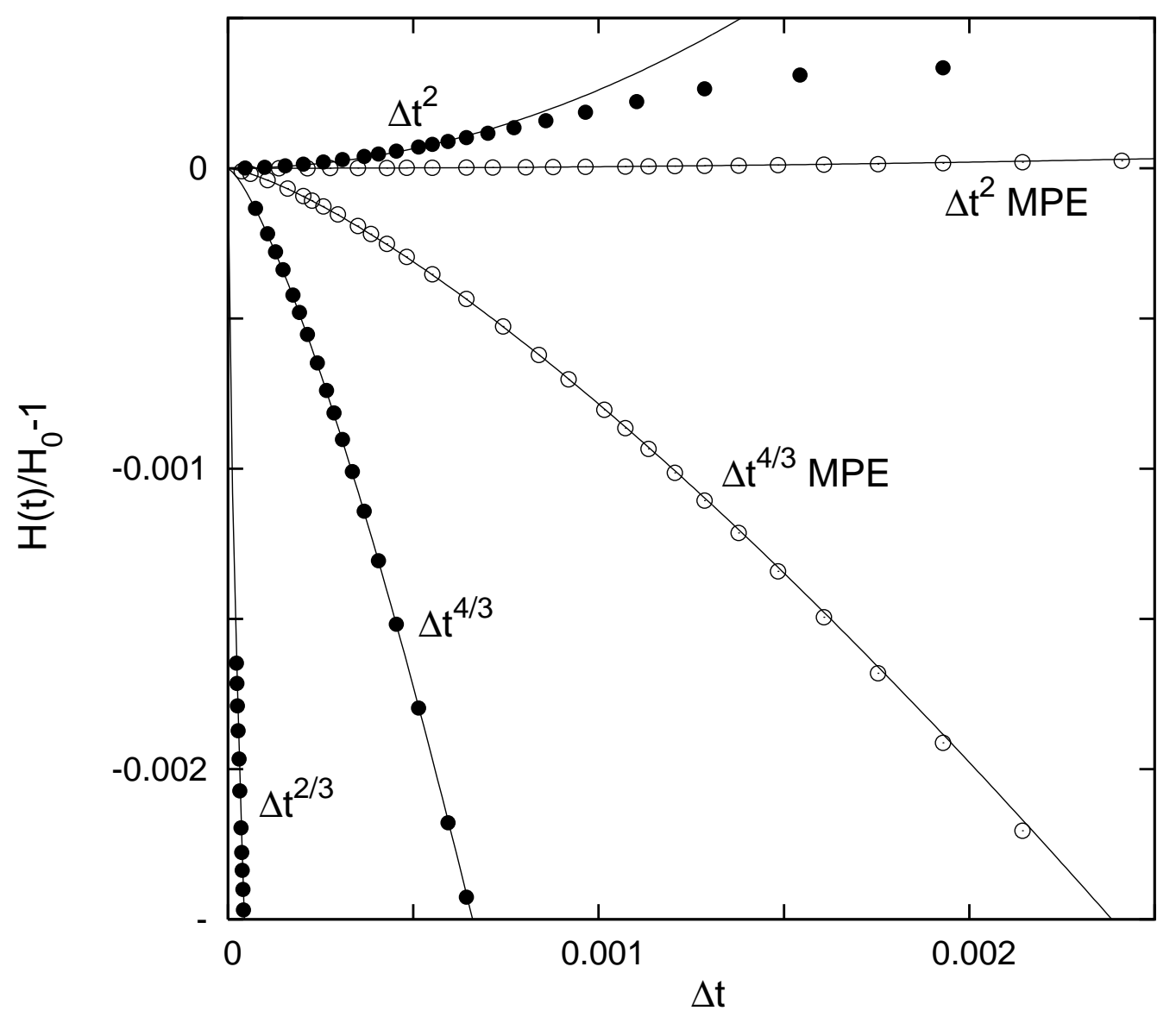

FIG. 3: The fractional power convergence of various explicit algorithms. The relative energy error is evaluated at the first quarter period $t=0.385841$, for the outermost trajectory of Fig.1. The solid circles denote results of symplectic algorithms (18), (30) and (31). The hallow circles give results of MPE algorithms (32) and (33). The lines are fitted cuves of the form $c \Delta t^{n}$, with $n=2 / 3,4 / 3$, or 2 as indicated. 